\title{
Neuronal Mitochondria Modulation of LPS-Induced Neuroinflammation
}

\author{
Micah Harland, ${ }^{1}$ Sandy Torres, ${ }^{1}$ Jingyi Liu, ${ }^{1}$ and Xinglong Wang ${ }^{1,2}$ \\ ${ }^{1}$ Department of Pathology, and ${ }^{2}$ Center for Mitochondrial Diseases, Case Western Reserve University, Cleveland, Ohio 44106
}

\begin{abstract}
Neuronal mitochondria dysfunction and neuroinflammation are two prominent pathological features increasingly realized as important pathogenic mechanisms for neurodegenerative diseases. However, little attempt has been taken to investigate the likely interactions between them. Mitofusin2 (Mfn2) is a mitochondrial outer membrane protein regulating mitochondrial fusion, a dynamic process essential for mitochondrial function. To explore the significance of neuronal mitochondria in the regulation of neuroinflammation, male and female transgenic mice with forced overexpression of Mfn2 specifically in neurons were intraperitoneally injected with lipopolysaccharide (LPS), a widely used approach to model neurodegeneration-associated neuroinflammation. Remarkably, LPS-induced lethality was almost completely abrogated in neuronal Mfn2 overexpression mice. Compared with nontransgenic wild-type mice, mice with neuronal Mfn2 overexpression also exhibited alleviated bodyweight loss, behavioral sickness, and myocardial dysfunction. LPS-induced release of IL- $1 \beta$ but not TNF- $\alpha$ was further found greatly inhibited in the CNS of mice with neuronal Mfn2 overexpression, whereas peripheral inflammatory responses in the blood, heart, lung, and spleen remained unchanged. At the cellular and molecular levels, neuronal Mfn2 suppressed the activation of microglia, prevented LPS-induced mitochondrial fragmentation in neurons, and importantly, upregulated the expression of CX3CL1, a unique chemokine constitutively produced by neurons to suppress microglial activation. Together, these results reveal an unrecognized possible role of neuronal mitochondria in the regulation of microglial activation, and propose neuronal Mfn2 as a likely mechanistic linker between neuronal mitochondria dysfunction and neuroinflammation in neurodegeneration.
\end{abstract}

Key words: LPS; Mfn2; mitochondrial dynamics; neuroinflammation; sepsis; septic myocardial dysfunction

\section{Significance Statement}

Our study suggests that Mfn2 in neurons contributes to the regulation of neuroinflammation. Based on the remarkable suppression of LPS-induced neuroinflammation and neurodegeneration-associated mitochondrial dysfunction and dynamic abnormalities by neuronal Mfn2, this study centered on Mfn2-mediated neuroinflammation reveals novel molecular mechanisms that are involved in both mitochondrial dysfunction and neuroinflammation in neurodegenerative diseases. The pharmacological targeting of Mfn2 may present a novel treatment for neuroinflammation-associated diseases.

\section{Introduction}

Progressive loss or dysfunction of neurons in the CNS or peripheral nervous system (PNS) is a characteristic feature of a wide range of neurodegenerative disorders including Alzheimer's disease (AD), Parkinson's disease, Huntington's disease, amyotrophic lateral sclerosis (ALS), and frontotemporal lobar degeneration. Although the cause that drives the progression of each of these neurodegenerative diseases remains elusive, it has been well recognized that these devastating diseases are multifactorial and

Received Sept. 27, 2019; revised Dec. 4, 2019; accepted Jan. 1, 2020.

Author contributions: X.W. designed research; M.H., S.T., and J.L. performed research; M.H. and X.W. wrote the paper.

This work was supported by Grants from the US NIH (1R01NS097679 and RF1AG056320) and U.S. Alzheimer's Association (AARG-17-499682).

The authors declare no competing financial interests.

Correspondence should be addressed to Xinglong Wang at xinglong.wang@case.edu.

https://doi.org/10.1523/JNEUROSCI.2324-19.2020

Copyright $@ 2020$ the authors involve many pathogenic mechanisms such as glutamate excitotoxicity, oxidative stress, neuroinflammation, and mitochondrial dysfunction in addition to the widely studied accumulation of misfolded or aggregated proteins. Among them, mitochondrial dysfunction and neuroinflammation have been extensively studied in the past decade. As prominent pathological features, both mitochondrial dysfunction and neuroinflammation are closely associated with pathological hallmarks (Heneka et al., 2015; Gao et al., 2017), and have been implicated as interdependent pathological lesions in neurodegenerative diseases (Wilkins and Swerdlow, 2016). However, despite intensive effort devoted to understanding the underlying cause(s) of these two prominent pathological features for neurodegenerative diseases, a clear mechanistic linker(s) between them has yet to be identified.

Mitofusin2 (Mfn2) is a conserved dynamin-like GTPase protein predominantly localized in the mitochondrial outer membrane regulating mitochondrial fusion (Chen et al., 2003), a process reported to be essential for various aspects of mitochon- 
drial function including respiratory complex assembly (Cogliati et al., 2013), ATP production (Benard et al., 2007), $\mathrm{Ca}^{2+}$ homeostasis (Frieden et al., 2004; Szabadkai et al., 2004), and reactive oxygen species production (Yu et al., 2006). Mfn2 has also been reported to be present in the endoplasmic reticulum (ER) or mitochondria-associated membranes to regulate ER and mitochondria tethering (de Brito and Scorrano, 2008; Sebastián et al., 2012; Sugiura et al., 2013), autophagosome formation (Hailey et al., 2010), autophagosome-lysosome fusion (Zhao et al., 2012), mitophagy (McLelland et al., 2018), and axonal transport of calpastatin, an endogenous specific inhibitor of the calpain system, to maintain neuromuscular synapses based on our most recent study (Wang et al., 2018). Altered mitochondrial dynamics (Kandimalla et al., 2016), distribution (Kopeikina et al., 2011), function (David et al., 2005), transport (RodríguezMartín et al., 2016), ER/mitochondria association (Perreault et al., 2009), autophagy (Schaeffer et al., 2012), and calpain (Reinecke et al., 2011), all Mfn2-related pathways, have been consistently observed in experimental models for neurodegenerative diseases.

Sepsis is a multi-symptomatic, life-threatening immune reaction to an infection. Although sepsis and neurodegenerative diseases are generally thought to be unrelated, both share similar neuroinflammation and neurologic symptoms (Sankowski et al., 2015). Additionally, a growing body of evidence supports that neuroinflammation and neurodegeneration can be initiated by robust inflammatory events in the periphery, including single dose lipopolysaccharide (LPS; Sheng et al., 2003; Kitazawa et al., 2005; Qin et al., 2007; Sy et al., 2011; Ifuku et al., 2012; Okuyama et al., 2013; Jin et al., 2014). Despite previous studies reporting altered mitochondrial dynamics in heart, lung, liver, kidney, and skeletal muscle tissue in sepsis animal models (Hansen et al., 2015; Liu et al., 2015; Yu et al., 2016; Park et al., 2018; Haileselassie et al., 2019; Tan et al., 2019), their role in the CNS during sepsis remains largely unknown. To examine this, we generated transgenic mice overexpressing Mfn2 specifically in CNS neurons under the control of Thy1.2 promotor, i.e., TMFN mice (Wang et al., 2015, 2018) and investigated the role of neuronal $\mathrm{Mfn} 2$ in neuroinflammation by intraperitoneally injecting TMFN and age-matched nontransgenic (NTg) mice with LPS, one of the most widely used approaches for peripherally induced neuroinflammation (Catorce and Gevorkian, 2016), and explored the potential pathways by which neuronal mitochondria regulate neuroinflammation via $\mathrm{Mfn} 2$.

\section{Materials and Methods}

Transgenic mice. All mouse procedures were performed in accordance with NIH guidelines and the Institutional Animal Care and Use Committee (IACUC) at Case Western Reserve University (CWRU). Mfn2 transgenic (TMFN) mice were created via pronuclear injection of the murine Thyl.2 genomic expression cassette (gift from Dr. Philip C. Wong, Johns Hopkins University) expressing human $M f n 2$ into C57BL/6 fertilized eggs. C57BL/6 Mfn $2^{f l / f l}$ mice were obtained from Dr. David Chan (California Institute of Technology). All experiments used 3-monthold male and female littermates raised in specific pathogen-free facilities. The animal number needed to reach statistical significance was calculated and reviewed by CWRU IACUC before the experiments.

LPS injection and survival. Three-month-old littermate mice were intraperitoneally injected with $10 \mathrm{mg} / \mathrm{kg}$ bodyweight of $0.22 \mu \mathrm{m}$ Millex filtered O111:B4 LPS (L2630, Sigma Aldrich) in PBS (in mM: $137 \mathrm{NaCl}, 2.7 \mathrm{KCl}, 10$ $\mathrm{Na}_{2} \mathrm{HPO}_{4}, 17.6 \mathrm{KH}_{2} \mathrm{PO}_{4}, \mathrm{pH} 7.4$ ) or an equal volume of PBS ipsilaterally. Survival was assessed daily up to $14 \mathrm{~d}$ postinjection (dpi), at which point remaining mice were killed in accordance with CWRU IACUC protocol.

Intrahippocampal injection. Three-month-old mice were intrahippocampally injected with $1 \mu \mathrm{l}$ of AAV1-shCX3CL1 and AAV1-
Scrambled control (TMFN and NTg mice) or AAV1-Cre-EGFP and AAV1-EGFP control ( $M f n 2^{f l / f l}$ mice; all from Vector Biolabs) on the left and right sides of the mice, respectively. Briefly, mice were anesthetized with $2 \%$ isoflurane inhalation and maintained at $2 \%$ via nosecone. A stereotactic platform with heating pad support was used to position the heads of the mice. Eye ointment was administered and the necks/heads of the mice were shaved before sterilization with betadine/alcohol. Bupivacaine/lidocaine $(1: 1 \mathrm{v} / \mathrm{v})$ was injected subcutaneously at the base of the neck. The skull was exposed with a scalpel midline incision from the frontal cranial bones to the parietal cranial bones. Small holes were drilled in the skull and AAV1 was injected ( $2.1 \mathrm{~mm}$ anteroposterior, \pm 2.0 $\mathrm{mm}$ mediolateral, and $-1.5 \mathrm{~mm}$ dorsoventral relative to bregma) with a 30 -gauge needle at a rate of $0.2 \mu \mathrm{l} / \mathrm{min}$ followed by 5 additional minutes for absorption. The incision was closed with nylon suture, carprofen was intraperitoneally administered, and mice were returned to a clean cage with warming pad for recovery. At $15 \mathrm{dpi}$, TMFN and NTg mice were intraperitoneally injected with $10 \mathrm{mg} / \mathrm{kg}$ LPS and killed $2 \mathrm{~d}$ later (17 dpi of AAV1). $M f n 2^{f l / f l}$ mice were killed at $21 \mathrm{dpi}$.

Microglia depletion. Three-month-old TMFN and NTg littermate mice were fed Purina Prolab RMH 3000 chow (5P75, Lab Diets) with or without 290 mg/kg PLX3397 (206178, MedKoo Biosciences) prepared by Lab Diets ad libitum for 4 weeks. Mice were intraperitoneally injected with 10 $\mathrm{mg} / \mathrm{kg}$ LPS or PBS control and remained on PLX3397 or control diet during the experiments.

Open-field tests. Open-field tests were conducted at 2 dpi during similar daytimes. A multiple unit open-field maze consisting of four chambers (50 cm length $\times 50 \mathrm{~cm}$ width $\times 38 \mathrm{~cm}$ height) was used for all tests. Each chamber was wiped with $10 \%$ ethanol before use and before subsequent tests to remove scent cues left by the previous mouse. Four lamps were placed at the outside corners of the test unit to allow for ample visibility within the chambers. Mice were acclimated in the chamber for $3 \mathrm{~min}$ before each trial and video recorded for $10 \mathrm{~min}$. Anymaze 6.13 software (Stoelting) was used to evaluate mouse movement/position (immobility settings at $65 \%$ and $2 \mathrm{~s}$ ). Inner zones were defined as a $40 \mathrm{~cm}$ length $\times 40 \mathrm{~cm}$ width square in the center of each chamber. Total traveled distance, immobile time, average speed, body rotations, and track plots were calculated using Anymaze 6.13.

Tissue collection. The majority of tissues and whole blood were collected at 2 dpi unless specified otherwise in the text. Briefly, mice were anesthetized with $2 \%$ isoflurane inhalation and killed via cervical dislocation for collection of non-perfused tissues. Perfused tissues, used where specified, were collected from mice anesthetized with Avertin (tribromoethanol, $2.5 \%$ in PBS) and transcardially perfused with $4^{\circ} \mathrm{C}$ PBS for $6 \mathrm{~min}$. Tissues for lysates were snap frozen on dry ice and stored at $-80^{\circ} \mathrm{C}$ before processing. Tissues for embedding were fixed in $10 \%$ formalin, dehydrated in increasing ethanol concentrations (70, 95, and $100 \%$ ), cleared in $100 \%$ xylene, and paraffinized in blocks.

Electrocardiogram. Electrocardiogram (ECG) recordings of mouse cardiac function was performed using the PowerLab 4/35 data acquisition system, FE136 Animal Bio Amp, and 29-G needle electrodes (ADInstruments). Mice were anesthetized with $2 \%$ isoflurane inhalation and maintained at $2 \%$ via nosecone. Sterile electrodes were inserted $\sim 1 \mathrm{~cm}$ subcutaneously proximal to each side of the thorax near the upper limbs and a reference grounding electrode was inserted subcutaneously near the left lower limb. ECGs were recorded with LabChart 8.1.13 software (ADInstruments) for 5 min with a 4000/s sampling rate. A low-pass filter with a cutoff frequency of $50 \mathrm{~Hz}$ was applied to improve signal-to-noise ratio. ECGs were block averaged to produce a single representative ECG and LabChart 8.1.13 software was used to automatically identify wave positions and amplitudes. Parameters were qualitatively validated against published mouse ECGs (Ho et al., 2011; Boukens et al., 2014) to ensure proper denotation of waves. Amplitude was measured as the voltage difference between $0 \mathrm{mV}$ baseline to the peak of each wave. Time intervals were measured as the time difference from the peak of one wave to another.

Hematology. Mouse tail-vein blood samples were collected in Microvette 200 lithium heparin capillary tubes (Sarsedt). White blood cell differentials were assessed with HEMAVET 950 hematology system (Drew Scientific). Plasma was prepared by centrifugation $\left(1000 \times g, 4^{\circ} \mathrm{C}\right)$ of tail vein blood for $10 \mathrm{~min}$ followed by collection of the clear top layer. 
Immunoblotting. Mouse tissues were lysed in $1 \times$ lysis buffer (9801, Cell Signaling Technology) with $1 \mathrm{~mm}$ phenylmethyl sulfonyl fluoride (10837091001, MilliporeSigma), Protease Inhibitor Cocktail (P8340, MilliporeSigma), and Phosphatase Inhibitor (4906845001, Roche). Protein concentrations were resolved using Pierce BCA Protein Assay (23227, ThermoFisher Scientific). Equal protein concentrations and volumes were run on $10 \%$ SDS-PAGE gels and blotted on Immobilon-P (IPVH00010, MilliporeSigma). Blots were blocked in 10\% nonfat milk in tris-buffered saline Tween (TBST; $50 \mathrm{~mm}$ Tris and $150 \mathrm{~mm} \mathrm{NaCl}, 0.1 \%$ Tween 20, $\mathrm{pH}$ 7.6) before probing with primary and detection antibodies in $1 \%$ milk in TBST. Blots were developed with Immobilon Western chemiluminescent horse radish peroxidase (HRP) substrate (WBKLS0500, MilliporeSigma) or ImmunoCruz Western Blotting Luminol Reagent (sc-2048, Santa Cruz Biotechnology) and imaged with ChemiDoc MP Imaging System (Bio-Rad).

Primary antibodies (all diluted 1:3000 in TBST from stock concentrations): mouse monoclonal anti-Drp1 (611112, BD Biosciences), rabbit monoclonal anti-GAPDH (2118, Cell Signaling Technology), mouse monoclonal anti- glial fibrillary acidic protein (GFAP; 14-9892-82, Invitrogen), mouse monoclonal anti-HIF- $1 \alpha$ (610958, BD Biosciences), rabbit monoclonal anti-Ibal (013-27691, Wako Chemical), rabbit polyclonal anti-MFF (12741, Abcam), mouse monoclonal anti-Mfn1 (sc166644, Santa Cruz Biotechnology), mouse monoclonal anti-Mfn2 (sc-100560, Santa Cruz Biotechnology), rabbit monoclonal anti-Mfn2 (11925, Cell Signaling Technology), mouse monoclonal anti-Opa1 (612606, BD Biosciences), rabbit polyclonal anti-MAP2 (AB5622, MilliporeSigma), rabbit monoclonal anti-Synaptophysin (5461S, Cell Signaling Technology), and mouse monoclonal anti- voltage-dependent anion channel 1 (VDAC1; ab14734, Abcam). Detection antibodies (diluted 1:10,000 in TBST from stock concentrations): anti-rabbit IgG, HRPlinked (7074S, Cell Signaling Technology), and anti-mouse IgG, HRPlinked (7076S, Cell Signaling Technology).

Immunohistochemistry (IHC). Formaldehyde-fixed paraffin embedded mouse tissue was used to prepare serial adjacent sections. Tissue sections were de-paraffinized with $100 \%$ xylene twice and rehydrated with decreasing ethanol concentrations $(100,95,70$, and 50\%) before incubation in tris-buffered saline (TBS; $50 \mathrm{~mm}$ Tris and $150 \mathrm{~mm} \mathrm{NaCl}, \mathrm{pH}$ 7.6). Tissue antigen retrieval was conducted with $1 \times$ immunoDNA retriever with citrate (BSB 0021, Bio SB) in a TintoRetriever pressure cooker (BSB 7008 , Bio SB). Slides were rinsed with deionized (DI) water and incubated with TBS. Individual sections were circled with hydrophobic marker and blocked with $10 \%$ normal goat serum (NGS; 50062Z, ThermoFisher Scientific) in TBS for $30 \mathrm{~min}$ at room temperature. Sections were rinsed with $1 \%$ NGS in TBS and excess liquid was removed with a paper towel. Sections were incubated with primary antibodies diluted in $1 \%$ NGS in TBS overnight at $4^{\circ} \mathrm{C}$, then rinsed and incubated with $1 \%$ followed by $10 \%$ NGS in TBS. Species-specific secondary antibodies were then placed on the tissue sections for $30 \mathrm{~min}$ at room temperature. Sections were rinsed and incubated in 1 and $10 \%$ NGS an additional time before incubation with species-specific peroxidase-anti-peroxidase for $1 \mathrm{~h}$ at room temperature. Slides were developed using a DAB chromogen kit (DB801L, Biocare Medical; or ENZ-ACC105, Enzo Life Sciences). Tris buffer ( 50 mM Tris, $\mathrm{pH} 7.6$ ) was used to stop the reaction and slides were rinsed with DI water and dehydrated with 70, 95, 100\% ethanol, and xylene. Coverslips were mounted with Permount (SP15-500, Fisher Scientific). Slides were imaged using a Zeiss Axio Imager.A2 equipped with an AxioCam 503 using Zeiss EC Plan-Neofluar $10 \times$ and $20 \times$ objectives.

For hematoxylin and eosin staining, tissue sections were rehydrated in decreasing ethanol concentrations as above and then placed in DI water. Slides were incubated in hematoxylin for $3 \mathrm{~min}$, rinsed in DI water, and excess stain removed with acid alcohol. Slides were thoroughly rinsed with DI water and incubated in eosin for $30 \mathrm{~s}$. Sections were dehydrated in 95 and $100 \%$ ethanol followed by xylene. Coverslips were mounted and slides were imaged as described for immunohistochemistry.

Primary antibodies (diluted in 1\% NGS in TBS from stock concentrations): mouse monoclonal anti-GFAP (1:250; 14-9892-82, Invitrogen), rabbit monoclonal anti-Ibal (1:250; 013-27691, Wako Chemical), rabbit monoclonal anti-Ki-67 (1:100; 9129S, Cell Signaling Technology), rabbit polyclonal anti-MAP2 (1:500; AB5622, MilliporeSigma), mouse mono- clonal anti-NeuN (1:1000; MAB377, MilliporeSigma), rabbit monoclonal anti-synaptophysin (1:100; 5461S, Cell Signaling Technology). Secondary and detection antibodies: goat anti-mouse IgG (1:50; AP124, MilliporeSigma), goat ant-rabbit IgG (1:50; AP132, MilliporeSigma), mouse peroxidase-anti-peroxidase (1:250; 223-005-024, Jackson ImmunoResearch), rabbit peroxidase-anti-peroxidase (1:250; 323-005-024, Jackson ImmunoResearch).

Immunofluorescent microscopy. Formaldehyde-fixed paraffin embedded mouse tissue was sectioned, de-paraffinized, rehydrated, and antigen retrieved. Slides were rinsed with DI water and incubated with PBS. Sections were circled with a hydrophobic marker and blocked with $10 \%$ NGS in PBS for 30 min. Sections were rinsed with $1 \%$ NGS in PBS and incubated with individual primary antibodies at $4^{\circ} \mathrm{C}$ overnight. Sections were then rinsed with $1 \%$ NGS, blocked in $10 \%$ NGS for $10 \mathrm{~min}$, and rinsed with $1 \%$ NGS. Sections were incubated with species-specific AlexaFluor 488- or 568-conjugated Abs diluted 1:300 in PBS for $2 \mathrm{~h}$ at room temperature in the dark. Sections were rinsed $3 \times$ with PBS, incubated with DAPI diluted 1:1000 in PBS for 15 min, and rinsed 3X with PBS. Excess liquid was removed and slides were coverslipped using Fluoromount-G mounting medium (0100-01, SouthernBiotech). Slides were imaged using a Zeiss Celldiscoverer 7 equipped with an AxioCam 512 and Hamamatsu Orca Flash 4.0 V3 using Zeiss Plan-Apochromat $20 \times$ and $50 \times$ autocorr objectives with $0.5 \times, 1 \times$, and $2 \times$ magnification changers.

Primary antibodies (diluted in 1\% NGS in PBS from stock concentrations): rabbit monoclonal anti-Ibal (1:250; 013-27691, Wako Chemical), mouse monoclonal anti-VDAC1 (1:3000; ab14734, Abcam). Detection antibodies: anti-mouse IgG (H+L) AlexaFluor 568 (1:300; A-11031, Invitrogen), anti-rabbit IgG (H+L) AlexaFluor 488 (1:300; A-11034, Invitrogen).

Enzyme-linked immunosorbent assay (ELISA). Optical 96-well plates were coated with polyclonal antigen capture antibody in ELISA Coating Buffer (421701, BioLegend) or, for CX3CL1 direct ELISA, $20 \mu \mathrm{g} / \mathrm{ml}$ mouse brain extract in PBS overnight at $4{ }^{\circ} \mathrm{C}$. Wells were aspirated and blocked with $1 \%$ bovine serum albumin (BSA) in PBS for $1.5 \mathrm{~h}$ at room temperature. Wells were aspirated, rinsed $3 \times$ with PBS, and aspirated. Recombinant protein standards and samples diluted in PBS were incubated in individual wells overnight at $4^{\circ} \mathrm{C}$. Wells were aspirated, rinsed with PBS $3 \times$, and aspirated. Primary antibody targeting the antigen of interest was diluted in PBS and added to each well. The plate was incubated at $4^{\circ} \mathrm{C}$ overnight. After aspirating, rinsing $3 \times$, and aspirating again, each well was incubated in diluted species-specific HRP-conjugate detection antibody for $2 \mathrm{~h}$ at room temperature. Wells were aspirated, rinsed $4 \times$, aspirated, and then developed with TMB substrate (N301, ThermoFisher Scientific) at $37^{\circ} \mathrm{C}$ for $30 \mathrm{~min} .450 \mathrm{~nm}$ stop solution (ab171529, Abcam) was added each well. The $\mathrm{OD}_{450}$ of each well was read with a BioTek 800TS microplate reader. Standard curves and protein concentrations were calculated from triplicate well averages in Microsoft Excel.

Capture antibodies (diluted in PBS): rabbit polyclonal anti-IL-1 $\beta$ (1 $\mu \mathrm{g} / \mathrm{ml}$; ab9722, Abcam), rabbit polyclonal anti-TNF $\alpha(0.5 \mu \mathrm{g} / \mathrm{ml}$; ab6671, Abcam). Primary antibodies: rabbit polyclonal anti-CX3CL1 (1 $\mu \mathrm{g} / \mathrm{ml}$; PA1-29026, Invitrogen), mouse monoclonal anti-IL-1 $\beta$ ( $1 \mu \mathrm{g} / \mathrm{ml}$; MAA563Mu21, Cloud-Clone), mouse monoclonal antiTNF $\alpha$ ( $1 \mu \mathrm{g} / \mathrm{ml}$; MAA133Mu21, Cloud-Clone). Detection antibodies (diluted 1:1000 in 1\% BSA in PBS from stock concentrations): antirabbit IgG, HRP-linked (7074S, Cell Signaling Technology), anti-mouse IgG, HRP-linked (7076S, Cell Signaling Technology). Protein standards: recombinant mouse IL-1 $\beta$ (ab78839, Abcam), recombinant mouse TNF $\alpha$ (ab9740, Abcam).

Real-time PCR. Total RNA was isolated from fresh perfused mouse whole brain tissue using RNeasy Mini kit (74104, Qiagen) according to the manufacturer's specifications. Complimentary DNA was synthesized using High-Capacity cDNA Reverse Transcription kit (4368814, Applied Biosystems) and Simpliamp Thermal Cycler (ThermoFisher Scientific) according to the manufacturer's cycling parameters. Real-time PCR for target mRNAs was assayed using Power SYBR Green Master Mix (4367660, Applied Biosystems) in a StepOne Real-Time PCR System (Life Technologies) according to the manufacturer's cycling parameters. StepOne 2.3 software (Life Technologies) was used to measure $C_{\mathrm{T}}$ values 
A
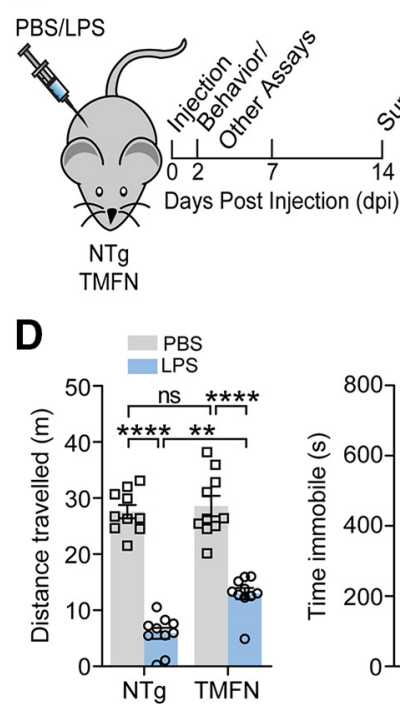

B
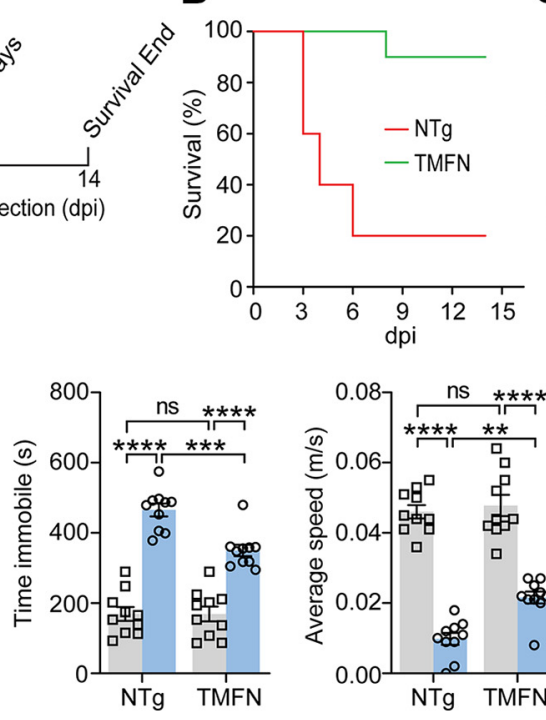

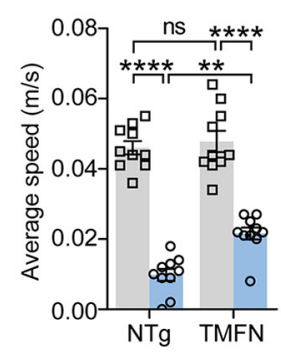

C

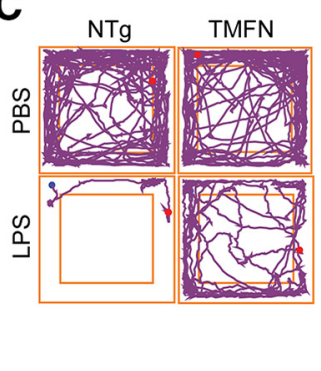

Results

TMFN mice are resistant to LPS-induced endotoxic shock

Mfn2 was only upregulated in the brain and spinal cord of TMFN mice and no transgene expression was noted in the peripheral immune system and other tissues, including the heart, lung, gastrocnemius muscle, liver and kidney (Fig. $1-1 A, B$, available at https://doi.org/10. 1523/JNEUROSCI.2324-19.2020.f1-1). Three-month-old TMFN and NTg littermates were intraperitoneally injected with a single lethal dose of LPS or PBS. Survival was monitored for up to $14 \mathrm{~d}$, whereas all other assays were conducted at $2 \mathrm{dpi}$ (Fig. $1 A)$. After intraperitoneal injection of lethal dose LPS, 3-month-old NTg mice showed body weight loss and died largely within 1 week (Fig. $1 B$ and Fig. 1-1C, available at https://doi.org/10.1523/ JNEUROSCI.2324-19.2020.f1-1). In striking contrast, TMFN mice demonstrated significantly alleviated body weight loss and most survived after lethal dose LPS challenge (Fig. 1-1C, available at https://doi.org/10.1523/ JNEUROSCI.2324-19.2020.f1-1), even though they also exhibited symptoms of acute illness. LPS-induced splenomegaly, a prominent feature reported in experimental sepsis models (Altamura et al., 2001), was similar in NTg and TMFN lit-

and fold-change standardized to GAPDH mRNA was calculated in Microsoft Excel.

The following primer pairs were used: CX3CL1 F: $5^{\prime}$-CGCGTTCTTC CATTTGTGTA-3' and R: 5'-CTGTGTCGTCTCCAGGACAA-3', CX3 CR1 F: 5'-CAGCATCGACCGGTACCTT-3' and R: 5'-GCTGCACT GTCCGGTTGTT-3', GAPDH F: 5'-ATGTTCCAGTATGACTCCAC TCACGG-3' and R: 5'-GAAGACACCAGTAGACTCCACGACA-3', IL- $1 \beta$ F: $5^{\prime}$-AACCTGCTGGTGTGTGACGTTC- $3^{\prime}$ and R: $5^{\prime}$-CAGCAC GAGGCTTTTTTGTT GT-3', IL-6 F: 5'-ACAACCACGGCCTTCCCT ACTT-3' and R: 5'-CACGATTTCCCAGAGAACATGTG-3', IL-10 F: 5'-ATAACTGCACCCACTTCCCA-3' and R: 5'-GGGCATCACTTC TACCA GGT-3', TNF $\alpha$ F: 5' -CTCCAGGCGGTGCCTATGT-3' and R: 5'-GAAGAGCGTGGTGGCCC-3'.

Image analysis. Immunoblot band densities were quantified with open-source WCIF ImageJ (developed by W. Rasband at the National Institutes of Health) and Image Lab 6.0.1 (Bio-Rad). IHC staining and immunofluorescent intensities were quantified with ZEN 2.3 (Carl Zeiss Microscopy). Immunofluorescent VDAC1 (AF568; green) and DAPI (blue) Z-stacks were processed for background subtraction and constrained iterative deconvolution before $3 \mathrm{D}$ projection in Zen software. Wide-field VDAC1 $Z$-stack images were similarly processed and mitochondrial length were calculated from single plane images using Zen 2.3 automated image analysis and Microsoft Excel. Mitochondrial length was defined as the Feret maximum $(F)$.

Experimental design and statistical analysis. Statistical analyses were conducted with Prism 8.0 (GraphPad). Data are mean \pm SEM. Data were compared by unpaired two-tailed $t$ tests for two samples or one-way ANOVA followed by Tukey's multiple comparison post hoc test for $>3$ samples. Sample size $(n)$ was defined as the number of cells counted in imaging experiments, or the number of mice per experimental group. The null hypothesis was rejected at the 0.05 level. $p$ values $<0.05$ were considered statistically significant. The statistical test, sample size $(n)$, and the $p$ values are all described in the figure legends. termates (Fig. 1-1D, available at https://doi.org/10.1523/ JNEUROSCI.2324-19.2020.f1-1). Weights of other tissues or organs were also identical, and showed similar pathological damage between NTg and TMFN littermates (Fig. 1-1D,E, available at https://doi.org/10.1523/JNEUROSCI.2324-19.2020.f1-1), suggesting that peripheral inflammatory responses and gross organ constitution are likely analogous in TMFN and NTg mice with LPS injection. Three independent lines of TMFN mice with similar transgene expression were tested and there was no phenotypic difference between them. Nonspecific psychological and behavioral symptoms, usually referred to as "sickness behavior", virtually accompany all acute inflammatory illnesses (Dantzer et al., 2008). To further investigate whether LPS-induced sickness behavior was improved in TMFN mice, we assessed the performance of mice in the open field test at 2 dpi. Compared with mice with PBS injection, NTg mice with LPS injection showed greatly reduced traveling distance and stayed largely immobile in proximity to the walls of the maze (Fig. 1C,D), indicative of both locomotor impairment and anxiety-related behavior. TMFN mice with LPS injection exhibited greater overall movement throughout the test with augmented distance traveled, reduced time spent immobile, and less wallhugging behavior (Fig. 1C,D). Together, these data demonstrate that forced expression of Mfn2 in neurons is sufficient to greatly alleviate LPS-induced endotoxic shock and associated behavioral sickness.

\section{LPS-induced neuroinflammation is attenuated in TMFN mice}

Peripheral LPS challenge causes widespread immune activation, including lasting neuroinflammation and neuronal dysfunction in mice (Catorce and Gevorkian, 2016). However, TMFN and NTg mice with the same injection type showed no differences in leukopenia and levels of plasma proinflammatory cytokines 
A

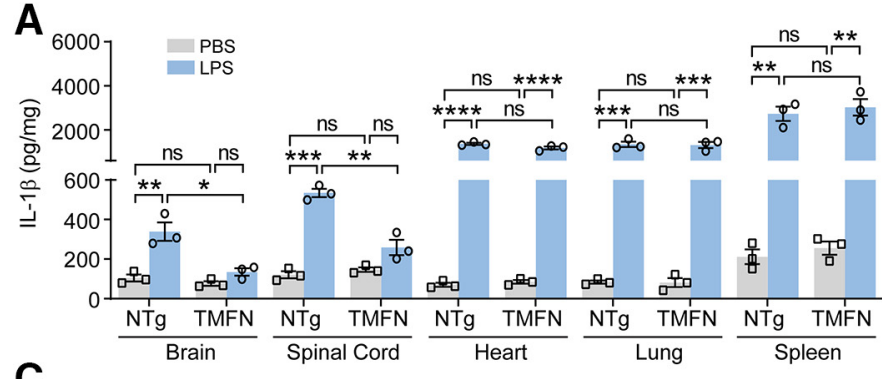

C

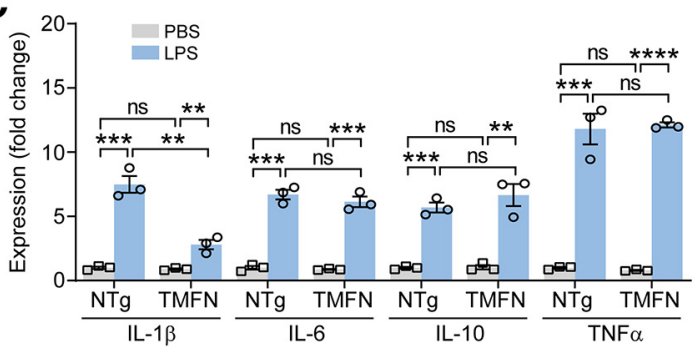

B

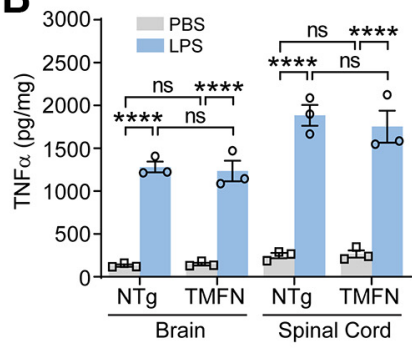

Figure 2. TMFN mice are protected from peripheral LPS-induced neuroinflammation. IL-1 $\beta(\boldsymbol{A})$ and TNF $\alpha(\boldsymbol{B})$ concentrations in the indicated tissues of TMFN and NTg mice at 2 dpi. Concentrations are per milligram of organ tissue ( $n=3$ mice/group). C, Quantification of IL-1 $\beta$, IL-6, IL-10, and TNF $\alpha$ mRNA levels in brains of TMFN and NTg mice at 2 dpi. Quantifications are normalized to GAPDH mRNA ( $n=3$ mice/group). Error bars represent mean \pm SEM, representative of triplicate experiments. Student's $t$ test or one-way ANOVA followed by Tukey's multiple-comparison test. ${ }^{*} p<0.05,{ }^{* *} p<0.01,{ }^{* * *} p<0.001,{ }^{* * * *} p<0.0001$. ns, Nonsignificant. For further details on peripheral inflammation see Figure 2-1, available at https://doi.org/10.1523/JNEUROSCI.232419.2020.f2-1. For the necessity of Mfn2 for neuroinflammation see Figure 2-2, available at https://doi.org/10.1523/JNEUROSCI.2324-19.2020.f2-2.

IL-1 $\beta$ and TNF $\alpha$ (Fig. 2-1A-C, available at https://doi.org/ 10.1523/JNEUROSCI.2324-19.2020.f2-1), further supporting that peripheral inflammation is unaltered in TMFN mice. Since plasma is specific to the circulatory system and does not constitute the entire immune environment, we then examined IL- $1 \beta$ and TNF $\alpha$ in multiple tissues from TMFN and NTg mice at $2 \mathrm{dpi}$, including the brain, spinal cord, heart, lung, and spleen. Consistent with their elevated plasma levels, both IL- $1 \beta$ and TNF $\alpha$ were greatly increased in these tissues of TMFN and NTg mice with LPS challenge (Fig. 2A,B). Surprisingly, although TNF $\alpha$ was similarly high in all surveyed tissues of LPS-treated TMFN and NTg mice, LPS-induced IL- $1 \beta$ increase was remarkably suppressed specifically in the brain and spinal cord of TMFN mice (Fig. $2 A, B$, and Fig. 2-1D, available at https://doi.org/10.1523/ JNEUROSCI.2324-19.2020.f2-1). Subsequent gene expression analyses validated the specific inhibition of IL- $1 \beta$ but not IL- 6 , IL-10, or TNF $\alpha$ at the mRNA level in the brains of TMFN mice with LPS injection compared with NTg mice with LPS (Fig. 2C). Of note, these cytokines were similar at basal levels in TMFN and NTg mice (Fig. 2A-C). These results indicate that forced Mfn2 expression in neurons attenuates peripherally induced neuroinflammation, likely through specific diminished induction of proinflammatory IL-1 $\beta$.

\section{LPS-induced microglia activation is suppressed in TMFN mice}

IL- $1 \beta$ is the main inflammatory mediator of the CNS and, when augmented, is commonly accompanied by microglia activation (Liu and Quan, 2018). Because TMFN mice displayed reduced IL- $1 \beta$ in the CNS following LPS injection, we next sought to determine whether microglia were differentially activated in TMFN mice. Brain sections from TMFN and NTg mice at 2 dpi were stained for ionized calcium binding adaptor molecule 1 (Iba1), a well established marker of microglia activation (Hoogland et al., 2015). Microglia of NTg mice with LPS injection exhibited fewer processes and increased Ibal staining, indicating microglia activation in the brain (Fig. $3 A, B$ ). In contrast,
TMFN microglia maintained ramified, inactive morphology with weak Iba1 staining after peripheral LPS challenge (Fig. $3 A, B$ ). Staining for GFAP, a marker of astrocyte activation, was increased to similar levels in the brains and spinal cords of TMFN and NTg mice with LPS injection (Fig. 3-1 A, B, available at https://doi.org/10.1523/JNEUROSCI.2324-19.2020.f3-1). To further quantify whether microglia were inactive in TMFN mice following LPS injection, we conducted immunofluorescent staining for Ibal in the brains of TMFN and NTg mice at 2 dpi. Microglia in NTg mice with LPS had rounded morphology with fewer processes that were shorter on average (Fig. $3 C, D$, and Fig. 3-1C, available at https://doi.org/10.1523/JNEUROSCI.232419.2020.f3-1). Contrastingly, microglia morphology, process number, and Ibal intensity were unaltered by LPS injection in TMFN mice with average process length only decreasing slightly compared with PBS injected control mice (Fig. 3C,D). To confirm that microglia were explicitly inactive in TMFN mice, we immunoblotted for Ibal and GFAP in brain extracts from TMFN and NTg mice at 2 dpi. Ibal levels were greatly increased in NTg mice with LPS injection, but only marginally increased in TMFN mice with LPS (Fig. 3-1D, available at https://doi.org/10.1523/ JNEUROSCI.2324-19.2020.f3-1). GFAP was increased equally in brains of TMFN and NTg mice with LPS injection (Fig. 3-1D, available at https://doi.org/10.1523/JNEUROSCI.2324-19.2020.f3-1), suggesting that microglia quiescence is likely driving reduced neuroinflammation in TMFN mouse brains.

\section{LPS-induced mitochondrial fragmentation in neurons is inhibited in TMFN mice}

To determine whether LPS could induce mitochondrial fragmentation in neurons of mice and whether forced Mfn2 expression could prevent this, mitochondrial morphology was assessed by immunofluorescent staining for mitochondria marker VDAC1 in brain sections from TMFN and NTg mice at 2 dpi. Compared with neurons of NTg mice, mitochondria in TMFN mouse neurons became elongated as evidenced by the increase in mitochondrial length; Fig. $4 A, B$, and Fig. $4-1 A, B$, available at 
A
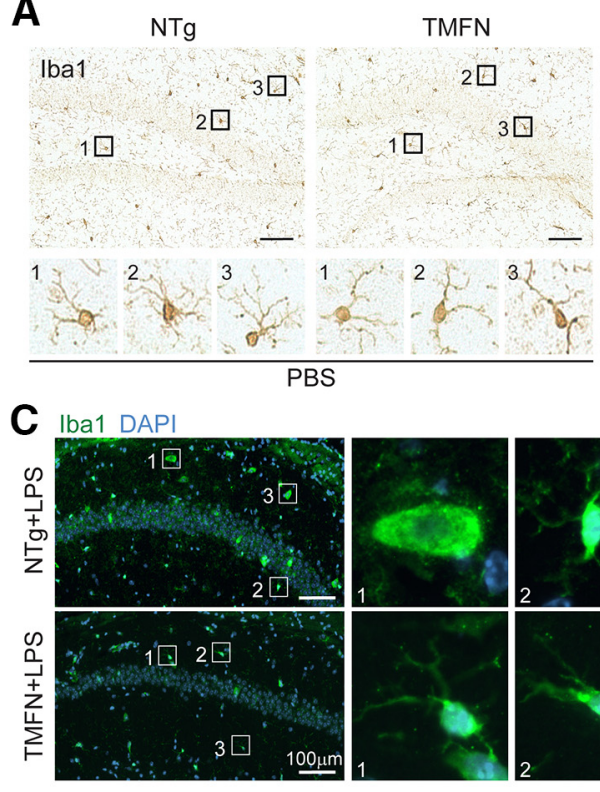

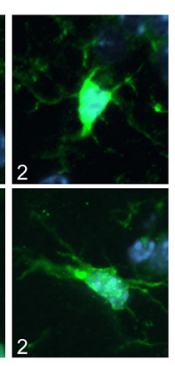

$\mathrm{NTg}$
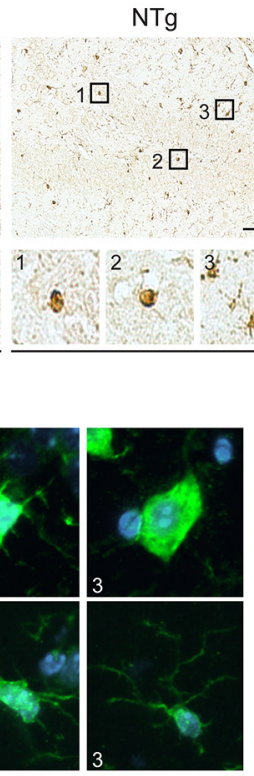

g
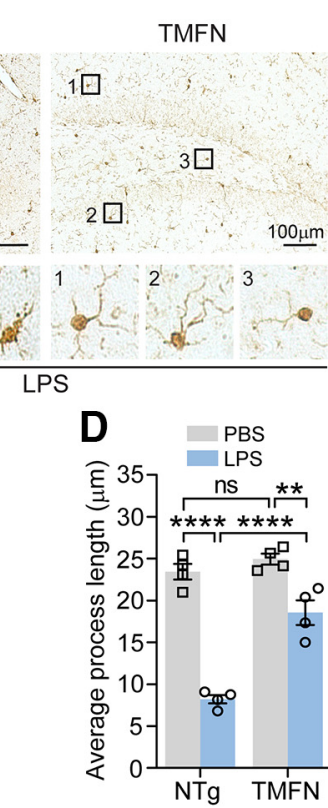
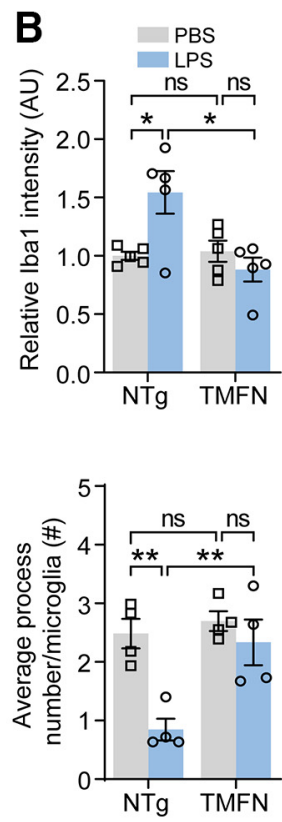

Figure 3. Microglia in TMFN mice are quiescent during peripheral LPS challenge. $A$, Representative IHC staining for Iba1 (microglia) in hippocampi of TMFN and NTg mice at $2 \mathrm{dpi}$. Enlarged images from boxed regions show individual microglia morphology ( $n=5$ mice/group). B, Quantification of lba1 intensity in frontal cortices of TMFN and NTg mice at 2 dpi from Iba1 IHC staining. AU, arbitrary units ( $n=5$ mice/group). C, Representative brain CA1 region immunofluorescent staining for lba1 (AF568; green) and nuclei (DAPI; blue) of TMFN and NTg mice at 2 dpi of LPS. Enlarged images from boxed regions show individual microglia morphology ( $n=4$ mice/group). D, Quantification of average microglia process length and process number from Iba1 (AF568) immunofluorescent staining of brains from TMFN and NTg mice at $2 \mathrm{dpi}$. The longest process on each microglia and number of processes per microglia were averaged from images of the hippocampus and frontal cortex for each mouse. Data points represent the average process lengths or process numbers for individual mice ( $n=4$ mice/group). Error bars represent mean \pm SEM, representative of triplicate experiments. Student's $t$ test or one-way ANOVA followed by Tukey's multiple-comparison test. ${ }^{*} p<0.05,{ }^{* *} p<0.01,{ }^{* * * *} p<0.0001$. ns, Nonsignificant. For further details on microglia and astrocyte activation see Figure 3-1, available at https://doi.org/10.1523/JNEUROSCI.2324-19.2020.f3-1. For the effect of microglia depletion see Figure 3-2, available at https://doi.org/10.1523/ JNEUROSCI.2324-19.2020.f3-2.

https://doi.org/10.1523/JNEUROSCI.2324-19.2020.f4-1, which was expected. In systemic LPS models, mitochondrial fragmentation has been demonstrated in multiple peripheral tissues (Gonzalez et al., 2014; Hansen et al., 2015; Haileselassie et al., 2019). Consistently, the significant reduction in mitochondrial length could also be noted in neurons of NTg mice with LPS injection (Fig. 4A,B). Mitochondria in TMFN neurons, however, still remained elongated after LPS challenge (Fig. 4A, B), demonstrating that peripheral LPS-induced mitochondrial fragmentation in neurons could be prevented by forced Mfn2 expression. We also investigated the expression of mitochondrial fission and fusion key regulators in brain and spinal cord tissues from TMFN and NTg mice with and without LPS injection. Immunoblot analysis confirmed significantly higher Mfn2 levels in TMFN mice. However, no significant changes in Mfn2 or other fusion regulators Mfn 1 and Opal were noted in LPS versus PBS-injected NTg or TMFN mice (Fig. 4-1C,D, available at https://doi.org/ 10.1523/JNEUROSCI.2324-19.2020.f4-1). Fission related proteins Drp1 and MFF were also unaltered (Fig. 4-1C,D, available at https://doi.org/10.1523/JNEUROSCI.2324-19.2020.f4-1), suggesting that mitochondrial fragmentation is unlikely because of the altered expression of fission and fusion regulators. Of note, overall mitochondrial contents were similar among all samples as evidenced by the constant expression of mitochondrial marker VDAC1 (Fig. 4-1C,D, available at https://doi.org/10.1523/ JNEUROSCI.2324-19.2020.f4-1). These data collectively implicate that peripheral inflammation can induce mitochondrial fragmentation in neurons and that increased fusion protein Mfn2 can alleviate fragmented mitochondrial morphology caused by LPS.

\section{Microglia activation inhibitor CX3CL1 is an indispensable} factor for Mfn2-mediated microglia inhibition

In the brain, CX3CL1 is produced by neurons and acts as the sole ligand of microglia expressed chemokine receptor CX3CR1 to mediate microglia activation in response to inflammatory stimuli such as LPS, IL-1 $\beta$, and TNF $\alpha$ (Zujovic et al., 2000, 2001; Mizuno et al., 2003; Biber et al., 2007). Although quantitative PCR (qPCR) analyses found the greatly increased level of CX3CL1 mRNA in the TMFN brains, CX3CR1 mRNA was found to be unchanged (Fig. 5A), implying a likely specific effect of neuronal Mfn2 on CX3CL1 transcription. Notably, LPS injection induced strong downregulation of CX3CL1 mRNA in the brains of NTg, which was not observed in TMFN mouse brains, whereas CX3CR1 mRNA expression was similar between genotypes and injection groups (Fig. 5A). Consistently, the protein level of CX3CL1 was greatly augmented in TMFN brains and remained at similarly high levels after LPS injection, even though NTg mice with LPS injection only exhibited a trend, but not statistically significant, toward reduced expression of CX3CL1 at the protein level in brains (Fig. 5B). We next virally knocked down CX3CL1 in the hippocampi of TMFN and NTg mice and injected peripheral LPS to determine whether CX3CL1 was critical for preventing microglia activation in TMFN mice and whether its knockdown could exacerbate neuroinflammation in LPSinjected NTg mice. Astonishingly, CX3CL1 knockdown restored microglia activation in the hippocampi of TMFN mice with LPS and augmented microglia activation in LPS-injected NTg mice as evidenced by increased Iba1/IL- $1 \beta$ staining and increased number of Ibal + microglia at the injection site compared with scrambled-control injected side (Fig. 5C,D, and Fig. 5-1, available 
A
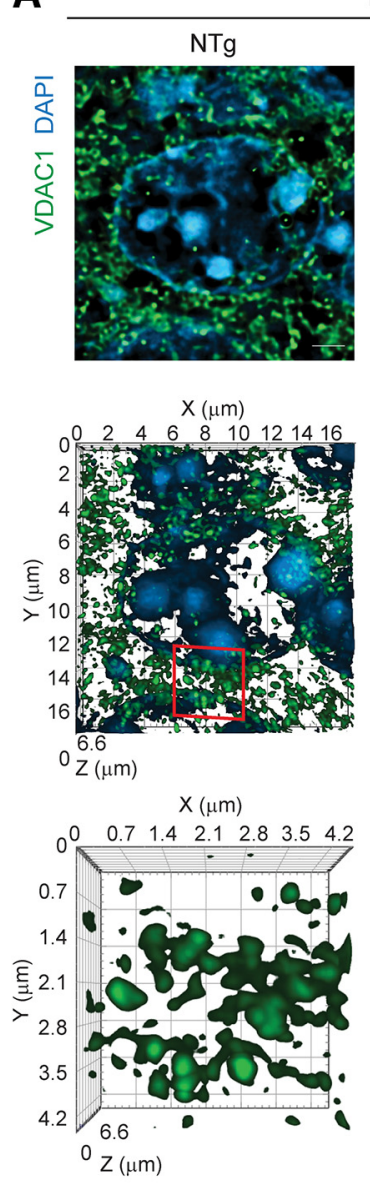

PBS
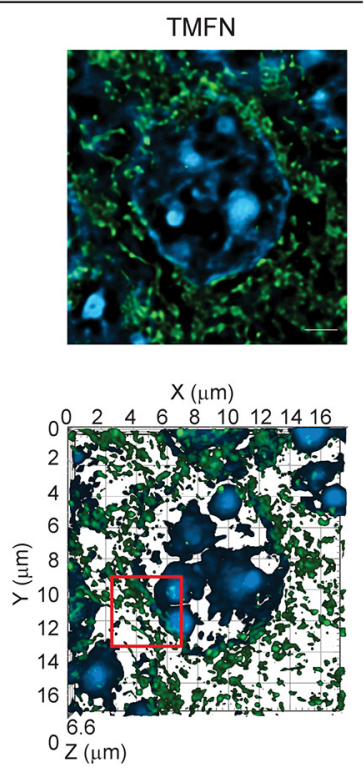

$X(\mu \mathrm{m})$

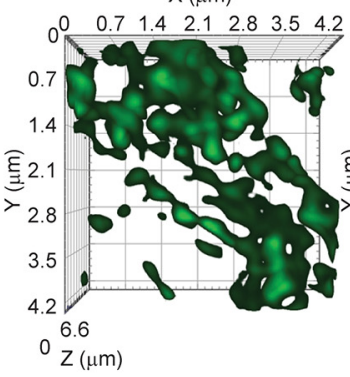

LPS

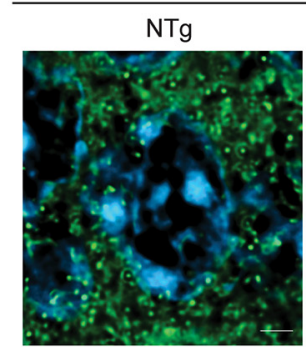

$X(u m)$

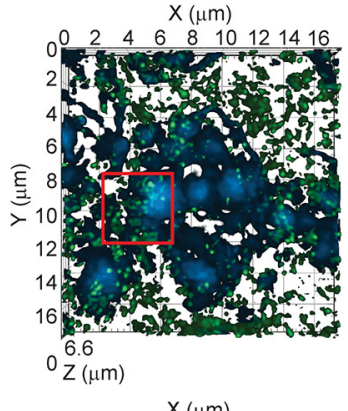

$\mathrm{X}(\mu \mathrm{m})$

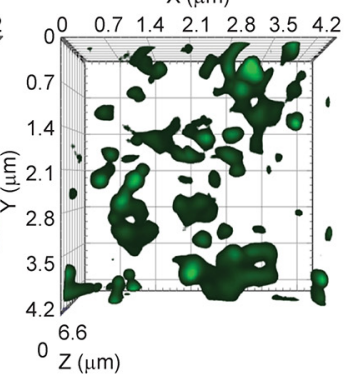

B

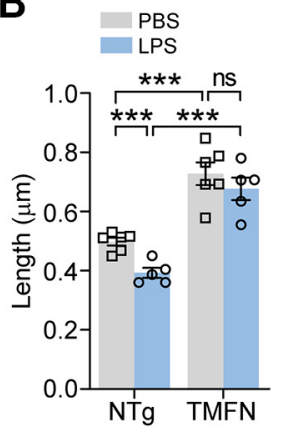

Figure 4. Mfn2 overexpression alleviates peripheral LPS-induced mitochondrial fragmentation in neurons. $A$, Representative single plane (top) and 3D $z$-stack illustrations (middle/bottom) of immunofluorescent staining for VDAC1 (AF488; green) and nuclei (DAPl; blue) in CA1 neurons of TMFN and NTg mice at 2 dpi. Red boxes indicate region enlarged in the bottom panels ( $n=4$ mice/group). B, Quantification of mitochondrial length in wide-field VDAC1 (AF488) immunofluorescent images of CA1 neurons of TMFN and NTg mice at 2 dpi. Measurements were averaged for individual CA1 neuronal images from each mouse. Data points represent the average mitochondrial length for individual mice (PBS injection: $n=6$ mice/group; LPS injection: $n=5$ mice/group). Error bars represent mean \pm SEM, representative of triplicate experiments. One-way ANOVA followed by Tukey's multiple-comparison test. ${ }^{* * *} p<0.001$. ns, Nonsignificant. For further details on mitochondrial dynamics see Figure 4-1, available at https://doi.org/10.1523/JNEUROSCI.2324-19.2020.f4-1. For neuronal viability see Figure 4-2, available at https://doi.org/10.1523/ JNEUROSCI.2324-19.2020.f4-2.

at https://doi.org/10.1523/JNEUROSCI.2324-19.2020.f5-1), supporting the necessity of CX3CL1 for Mfn2-mediated microglia inhibition. While further mechanistic insight is needed to identify how mitochondrial dynamics or Mfn2 expression regulate CX3CL1 expression in neurons, these data suggest that forced Mfn2 expression in neurons drives expression of microglia inhibitory CX3CL1, potentially blocking peripheral-induced neuroinflammation.

\section{Discussion}

In this study we demonstrate for the first time that genetic upregulation of Mfn2 in neurons is sufficient to remarkably prevent sepsis lethality and sickness behavior induced by peripheral injection of lethal dose LPS. Interestingly, neuronal Mfn2 overexpression resulted in the specific inhibition of microglia but not astrocytes. Likewise, LPS-induced IL- $1 \beta$ but not TNF $\alpha$, IL-6, or IL-10 increase was also specifically suppressed by neuronal Mfn2. Because microglial inhibitor CX3CL1 produced by neurons was found greatly upregulated by neuronal Mfn2, Mfn2 likely acts as an unrecognized neuroinflammatory modulator in neurons. Neuronal Mfn2 ablation alone induces IL-1 $\beta$ release (Fig. 2-2A-C, available at https://doi.org/10.1523/JNEUROSCI.2324- 19.2020.f2-2), implying its necessity for neuroinflammation. Notably, Mfn2 is reduced in patients with AD or ALS (Wang et al., 2009, 2018). Therefore, neuronal Mfn2 may be a point of convergence for neuronal mitochondria dysfunction and neuroinflammation in neurodegeneration, worthy of further detailed investigation.

One of the most important and surprising findings of this study was that upregulation of Mfn2 in neurons was associated with almost completely abolished mortality in mice injected with a lethal dose of LPS. Mfn2 is only upregulated in the brain and spinal cord of TMFN mice. Even though the Thyl promoter can drive transgene expression in enteric neurons (Goto et al., 2013), TMFN mouse peripheral tissues did not show increased Mfn2 expression (Fig. 1-1 A, B, available at https://doi.org/10.1523/ JNEUROSCI.2324-19.2020.f1-1). On top of these, TMFN mice exhibited LPS-induced peripheral inflammatory responses similar to NTg mice, together excluding the involvement of peripheral immune system in the resistance of TMFN mice to LPSinduced lethality. Cardiac dysfunction is a prominent feature of septic shock in response to infection and has been implicated as an important component contributing to its high mortality $(\mathrm{Ru}-$ 
A

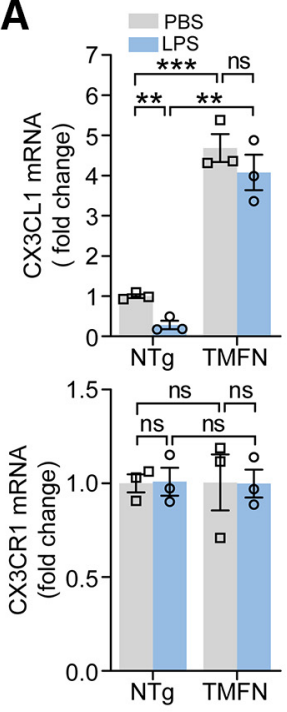

B

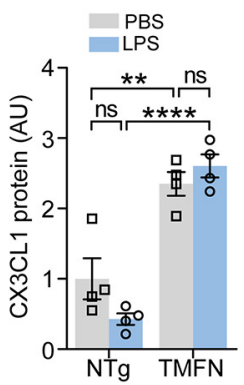

C

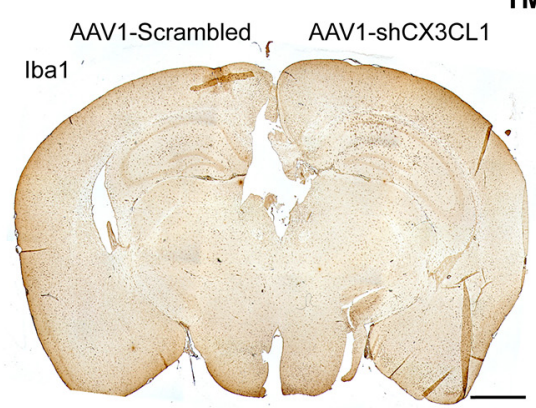

TMFN
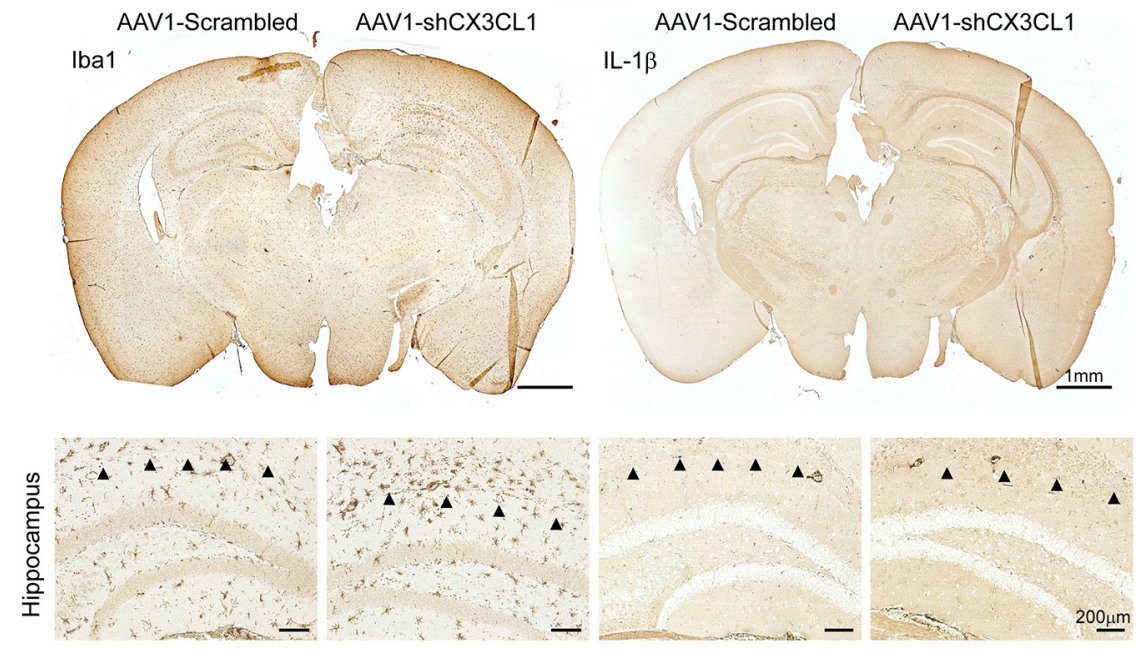

D AAV1-Scrambled
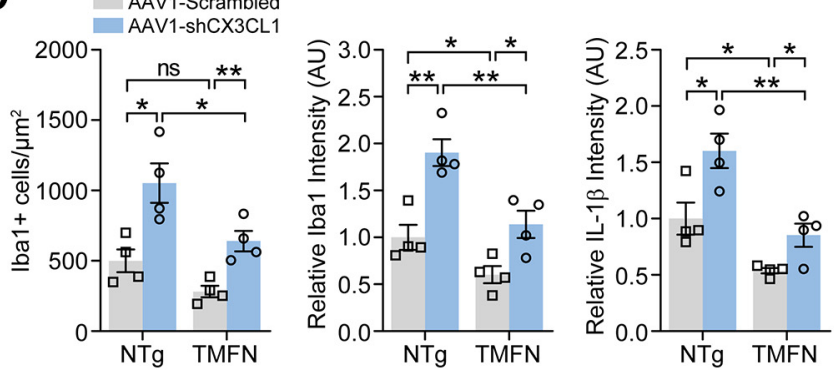

Figure 5. $\quad$ XX3 $\mathrm{CL} 1$ is upregulated and required for microglia inhibition in TMFN mice. $A, C X 3 C \mathrm{CL} 1$ (top) and $C X 3 C \mathrm{C} 1$ (bottom) mRNA levels in brains of TMFN and NTg mice at $2 \mathrm{dpi}$ were determined by qPCR. Fold changes were standardized to GAPDH mRNA for each mouse ( $n=3$ mice/group). $B, C X 3 C L 1$ protein levels in brains of TMFN and NTg mice at 2 dpi were assessed by ELISA. AU, Arbitrary units ( $n=4$ mice/group). C, Representative whole brain and hippocampus IHC staining for Iba1 and IL-1 $\beta$ in LPS-injected TMFN mice with intrahippocampal AAV1-shCX3CL1 (right) and AAV1-Scrambled (left, control). Brains were isolated from TMFN and NTg mice 17 dpi of AAV1 and 2 dpi LPS. Arrowheads show hippocampal injection sites with increased Iba1 + cell number and IL-1 $\beta$ staining in AAV1-shCX3CL1-injected mice ( $n=4$ mice/group). D, Quantifications from IHC of Iba1 + cell count (left), Iba1 intensity (middle), and IL-1 $\beta$ intensity (right) in hippocampal injection sites of TMFN and NTg mice from C ( $n=4$ mice/group). Error bars represent mean \pm SEM, representative of triplicate experiments. Student's $s$ test and one-way ANOVA followed by Tukey's multiple-comparison test. ${ }^{*} p<0.05,{ }^{* *} p<0.01,{ }^{* * *} p<0.001$, ${ }^{* * * *} p<0.0001$. ns, Nonsignificant. For further details on CX3CL1 ablation see Figure 5-1, available at https://doi.org/ 10.1523/JNEUROSCI.2324-19.2020.55-1.

diger and Singer, 2007). Although inflammatory cytokine release and Mfn2 expression were similar in NTg and TMFN heart tissues, there was clear absence of ischemic QT depression and a decrease in myocardial ischemia and infarction indicators HIF- $1 \alpha$ and Ki-67 in TMFN mice (Fig. 1-2A-F, available at https://doi.org/10.1523/JNEUROSCI.2324-19.2020.f1-2), indicating that neuronal Mfn2-regulated cardiac function, potentially through autonomic regulation, is likely an important mechanism for the suppression of lethality associated with septic shock in TMFN mice. In support of this notion, previous studies have identified autonomic control of myocardial dysfunction in LPS rodent models, particularly through cholinergic neurons of the sympathovagal system (Plaschke et al., 2018; Sallam et al., 2018; Ndongson-Dongmo et al., 2019). Nevertheless, additional work is still needed to clarify whether autonomic neurotransmission is altered by Mfn2 overexpression in neurons. LPS has been reported to severely affect neural activity and dopamine release in reward-related brain regions in association with reduced motivation and anhedonia (Felger et al., 2016). Accordingly, despite the absence of neuronal loss or altered expression of neuronal markers NeuN, MAP2, and synaptophysin in our results (Fig. 4-2A-D, available at https://doi.org/10.1523/JNEUROSCI.232419.2020.f4-2), mitigated LPS-induced sickness behavior was noted in TMFN mice. Therefore, Mfn2 may regulate neural activity and dopamine release in preventing the development of cognitive and behavioral complications, worthy of further exploration. In addition to heart injury, systematic ischemic organ damage is present in murine LPS models (Cecconi et al., 2018). Because TMFN animals challenged with LPS displayed significantly alleviated bodyweight loss, future studies will also be interesting to investigate the possible Mfn2-mediated autonomic protection of other peripheral organs and tissues, which are known to be tightly controlled by the CNS and PNS.

Another interesting finding of the present study was the specific inhibition of LPS-induced IL-1 $\beta$ but not IL-6, IL-10, or TNF $\alpha$ increase in the CNS by forced overexpression of neuronal Mfn2. IL- $1 \beta$ is a proinflammatory cytokine that is upregulated in $\mathrm{AD}$ and many neurodegenerative diseases, and believed to be the key player driving the neuroinflammatory process (Shaftel et al., 2008). By binding to its cognate receptor, IL- $1 \beta$ initiates a signaling cascade through the type I IL- 1 receptor to activate NF- $\kappa \mathrm{B}$ dependent transcription of inflammatory gene expression (Weber et al., 2010). The majority of IL-1 $\beta$ research has been conducted on peripheral cells, but it appears that similar signaling mechanisms could be involved in IL- $1 \beta$-mediated neuroinflammation in CNS cells. IL- $1 \beta$ and other members of the IL- 1 family can be constitutively synthesized by neural cells in the brain and have long been implied as neuromodulators in addition to proinflammatory factors (Vitkovic et al., 2000). Although the specific cellular source and the regulation mechanisms for 
expression, cleavage, and release remain largely elusive, activated microglia are generally believed the to be the primary source of IL- $1 \beta$ in the brain (Rothwell and Luheshi, 2000). In support of this notion, microglia depletion was found to suppress LPS-induced locomotive deficits (Fig. 3-2A-D, available at https://doi.org/10.1523/ JNEUROSCI.2324-19.2020.f3-2). Indeed, TMFN mice demonstrated greatly abrogated basal microglia activation. Furthermore, despite the fact that astrocytes also play a critical role in regulating neuroinflammation and that IL- $1 \beta$ alone is capable of eliciting astrocyte and microglia activation in the rodent brain (Rothwell and Luheshi, 2000; Felger et al., 2016), neuronal Mfn2 appears to have no effect on basal or LPS-induced astrocyte activation. Therefore, our results indicate that neuronal Mfn2 may control IL- $1 \beta$ expression or release by specifically participating in microglia activation.

CX3CR1 and its sole ligand CX3CL1 are critical mediators between neurons and microglia in neurodegenerative diseases (Finneran and Nash, 2019). Despite the dependence of neuroprotective or neurotoxic functions of the CX3CL1/CX3CR1 signaling pathway on microglial activation stimuli or pathological condition, a large body of evidence has implicated CX3CL1 as a potent inhibitor of microglial activation (Biber et al., 2007). Excitingly, we found significantly increased expression of CX3CL1 but not CX3CR1 in the CNS of TMFN mice. Knockdown of CX3CL1 was also sufficient to restore microglia activation and IL- $1 \beta$ release in hippocampi of LPS-injected TMFN mice. Therefore, a plausible mechanistic explanation for the specific inhibitory effects of neuronal Mfn 2 on microglial activation and related cytokine release is that Mfn2 in neurons might contribute to the regulation of CX3CL1/CX3CR1 signaling by modulating the expression of CX3CL1. As a predominant mitochondrial outer membrane protein, Mfn2 unlikely regulates CX3CL1 expression or transcription directly. Since LPS-induced mitochondrial fragmentation was alleviated in TMFN mice, it is highly possible that neuronal Mfn2 may regulate CX3CL1 expression via mitochondrial dynamics-dependent metabolism or the transport of neuronal factors required for CX3CL1 expression. However, considering the functional role of Mfn2 in ER and mitochondria tethering, autophagy, and calpastatin transport, we could not exclude the possibility that Mfn2 acts via other pathways to regulate neuroinflammation via CX3CL1. Future detailed investigation of the dependence of Mfn2-mediated CX3CL1 expression on mitochondrial fission and fusion dynamics and the sufficiency of mitochondrial fission and fusion dynamics for CX3CL1 regulation will provide a clear answer.

Together, these data suggest that Mfn2 in neurons contributes to the regulation of neuroinflammation. Although mitochondrial dysfunction and neuroinflammation are two prominent pathological features of neurodegenerative diseases, whether and how neuronal mitochondria and neuroinflammation are interrelated is largely unknown. Based on the remarkable suppression of LPS-induced neuroinflammation and neurodegenerationassociated mitochondrial dysfunction and dynamic abnormalities by neuronal Mfn2 (Wang et al., 2011, 2012, 2018), this study centered on Mfn2-mediated neuroinflammation reveals novel molecular mechanisms that are involved in both mitochondrial dysfunction and neuroinflammation in neurodegenerative diseases. The pharmacological targeting of Mfn2 may present a novel treatment for neuroinflammation-associated diseases.

\section{References}

Altamura M, Caradonna L, Amati L, Pellegrino NM, Urgesi G, Miniello S (2001) Splenectomy and sepsis: the role of the spleen in the immunemediated bacterial clearance. Immunopharmacol Immunotoxicol 23: 153-161.
Benard G, Bellance N, James D, Parrone P, Fernandez H, Letellier T, Rossignol R (2007) Mitochondrial bioenergetics and structural network organization. J Cell Sci 120:838-848.

Biber K, Neumann H, Inoue K, Boddeke HW (2007) Neuronal “on" and "off” signals control microglia. Trends Neurosci 30:596-602.

Boukens BJ, Rivaud MR, Rentschler S, Coronel R (2014) Misinterpretation of the mouse ECG: "musing the waves of Mus musculus". J Physiol 592:4613-4626.

Catorce MN, Gevorkian G (2016) LPS-induced murine neuroinflammation model: main features and suitability for pre-clinical assessment of nutraceuticals. Curr Neuropharmacol 14:155-164.

Cecconi M, Evans L, Levy M, Rhodes A (2018) Sepsis and septic shock. Lancet 392:75-87.

Chen H, Detmer SA, Ewald AJ, Griffin EE, Fraser SE, Chan DC (2003) Mitofusins Mfn1 and Mfn2 coordinately regulate mitochondrial fusion and are essential for embryonic development. J Cell Biol 160:189-200.

Cogliati S, Frezza C, Soriano ME, Varanita T, Quintana-Cabrera R, Corrado M, Cipolat S, Costa V, Casarin A, Gomes LC, Perales-Clemente E, Salviati L, Fernandez-Silva P, Enriquez JA, Scorrano L (2013) Mitochondrial cristae shape determines respiratory chain supercomplexes assembly and respiratory efficiency. Cell 155:160-171.

Dantzer R, O'Connor JC, Freund GG, Johnson RW, Kelley KW (2008) From inflammation to sickness and depression: when the immune system subjugates the brain. Nat Rev Neurosci 9:46-56.

David DC, Hauptmann S, Scherping I, Schuessel K, Keil U, Rizzu P, Ravid R, Drose S, Brandt U, Müller WE, Eckert A, Götz J (2005) Proteomic and functional analyses reveal a mitochondrial dysfunction in P301L tau transgenic mice. J Biol Chem 280:23802-23814.

de Brito OM, Scorrano L (2008) Mitofusin 2 tethers endoplasmic reticulum to mitochondria. Nature 456:605-610.

Felger JC, Li Z, Haroon E, Woolwine BJ, Jung MY, Hu X, Miller AH (2016) Inflammation is associated with decreased functional connectivity within corticostriatal reward circuitry in depression. Mol Psychiatry 21:13581365.

Finneran DJ, Nash KR (2019) Neuroinflammation and fractalkine signaling in Alzheimer's disease. J Neuroinflammation 16:30.

Frieden M, James D, Castelbou C, Danckaert A, Martinou JC, Demaurex N (2004) $\mathrm{Ca}^{2+}$ homeostasis during mitochondrial fragmentation and perinuclear clustering induced by hFis1. J Biol Chem 279:22704-22714.

Gao J, Wang L, Liu J, Xie F, Su B, Wang X (2017) Abnormalities of mitochondrial dynamics in neurodegenerative diseases. Antioxidants 6:E25.

Gonzalez AS, Elguero ME, Finocchietto P, Holod S, Romorini L, Miriuka SG, Peralta JG, Poderoso JJ, Carreras MC (2014) Abnormal mitochondrial fusion-fission balance contributes to the progression of experimental sepsis. Free Radic Res 48:769-783.

Goto K, Kato G, Kawahara I, Luo Y, Obata K, Misawa H, Ishikawa T, Kuniyasu H, Nabekura J, Takaki M (2013) In vivo imaging of enteric neurogenesis in the deep tissue of mouse small intestine. PLoS One 8:e54814.

Haileselassie B, Mukherjee R, Joshi AU, Napier BA, Massis LM, Ostberg NP, Queliconi BB, Monack D, Bernstein D, Mochly-Rosen D (2019) Drp1/ Fis1 interaction mediates mitochondrial dysfunction in septic cardiomyopathy. J Mol Cell Cardiol 130:160-169.

Hailey DW, Rambold AS, Satpute-Krishnan P, Mitra K, Sougrat R, Kim PK, Lippincott-Schwartz J (2010) Mitochondria supply membranes for autophagosome biogenesis during starvation. Cell 141:656-667.

Hansen ME, Simmons KJ, Tippetts TS, Thatcher MO, Saito RR, Hubbard ST, Trumbull AM, Parker BA, Taylor OJ, Bikman BT (2015) Lipopolysaccharide disrupts mitochondrial physiology in skeletal muscle via disparate effects on sphingolipid metabolism. Shock 44:585-592.

Heneka MT, Carson MJ, El Khoury J, Landreth GE, Brosseron F, Feinstein DL, Jacobs AH, Wyss-Coray T, Vitorica J, Ransohoff RM, Herrup K, Frautschy SA, Finsen B, Brown GC, Verkhratsky A, Yamanaka K, Koistinaho J, Latz E, Halle A, Petzold GC, et al. (2015) Neuroinflammation in Alzheimer's disease. Lancet Neurol 14:388-405.

Ho D, Zhao X, Gao S, Hong C, Vatner DE, Vatner SF (2011) Heart rate and electrocardiography monitoring in mice. Curr Protoc Mouse Biol 1:123139.

Hoogland IC, Houbolt C, van Westerloo DJ, van Gool WA, van de Beek D (2015) Systemic inflammation and microglial activation: systematic review of animal experiments. J Neuroinflammation 12:114.

Ifuku M, Katafuchi T, Mawatari S, Noda M, Miake K, Sugiyama M, Fujino T (2012) Anti-inflammatory/anti-amyloidogenic effects of plasmalogens 
in lipopolysaccharide-induced neuroinflammation in adult mice. J Neuroinflammation 9:197.

Jin M, Jang E, Suk K (2014) Lipocalin-2 acts as a neuroinflammatogen in lipopolysaccharide-injected mice. Exp Neurobiol 23:155-162.

Kandimalla R, Manczak M, Fry D, Suneetha Y, Sesaki H, Reddy PH (2016) Reduced dynamin-related protein 1 protects against phosphorylated tauinduced mitochondrial dysfunction and synaptic damage in Alzheimer's disease. Hum Mol Genet 25:4881-4897.

Kitazawa M, Oddo S, Yamasaki TR, Green KN, LaFerla FM (2005) Lipopolysaccharide-induced inflammation exacerbates tau pathology by a cyclin-dependent kinase 5-mediated pathway in a transgenic model of Alzheimer's disease. J Neurosci 25:8843-8853.

Kopeikina KJ, Carlson GA, Pitstick R, Ludvigson AE, Peters A, Luebke JI, Koffie RM, Frosch MP, Hyman BT, Spires-Jones TL (2011) Tau accumulation causes mitochondrial distribution deficits in neurons in a mouse model of tauopathy and in human Alzheimer's disease brain. Am J Pathol 179:2071-2082.

Liu L, Song Y, Zhao M, Yi Z, Zeng Q (2015) Protective effects of edaravone, a free radical scavenger, on lipopolysaccharide-induced acute kidney injury in a rat model of sepsis. Int Urol Nephrol 47:1745-1752.

Liu X, Quan N (2018) Microglia and CNS interleukin-1: beyond immunological concepts. Front Neurol 9:8.

McLelland GL, Goiran T, Yi W, Dorval G, Chen CX, Lauinger ND, Krahn AI, Valimehr S, Rakovic A, Rouiller I, Durcan TM, Trempe JF, Fon EA (2018) Mfn2 ubiquitination by PINK1/parkin gates the p97-dependent release of ER from mitochondria to drive mitophagy. eLife 7:e32866.

Mizuno T, Kawanokuchi J, Numata K, Suzumura A (2003) Production and neuroprotective functions of fractalkine in the central nervous system. Brain Res 979:65-70.

Ndongson-Dongmo B, Lang GP, Mece O, Hechaichi N, Lajqi T, Hoyer D, Brodhun M, Heller R, Wetzker R, Franz M, Levy FO, Bauer R (2019) Reduced ambient temperature exacerbates SIRS-induced cardiac autonomic dysregulation and myocardial dysfunction in mice. Basic Res Cardiol 114:26.

Okuyama S, Makihata N, Yoshimura M, Amakura Y, Yoshida T, Nakajima M, Furukawa Y (2013) Oenothein B suppresses lipopolysaccharide (LPS)induced inflammation in the mouse brain. Int J Mol Sci 14:9767-9778.

Park JS, Choi HS, Yim SY, Lee SM (2018) Heme oxygenase-1 protects the liver from septic injury by modulating TLR4-mediated mitochondrial quality control in mice. Shock 50:209-218.

Perreault S, Bousquet O, Lauzon M, Paiement J, Leclerc N (2009) Increased association between rough endoplasmic reticulum membranes and mitochondria in transgenic mice that express P301 L tau. J Neuropath Exp Neurol 68:503-514.

Plaschke K, Do TQM, Uhle F, Brenner T, Weigand MA, Kopitz J (2018) Ablation of the right cardiac vagus nerve reduces acetylcholine content without changing the inflammatory response during endotoxemia. Int J Mol Sci 19:E442.

Qin L, Wu X, Block ML, Liu Y, Breese GR, Hong JS, Knapp DJ, Crews FT (2007) Systemic LPS causes chronic neuroinflammation and progressive neurodegeneration. Glia 55:453-462.

Reinecke JB, DeVos SL, McGrath JP, Shepard AM, Goncharoff DK, Tait DN, Fleming SR, Vincent MP, Steinhilb ML (2011) Implicating calpain in tau-mediated toxicity in vivo. Plos One 6:e23865.

Rodríguez-Martín T, Pooler AM, Lau DHW, Morotz GM, De Vos KJ, Gilley J, Coleman MP, Hanger DP (2016) Reduced number of axonal mitochondria and tau hypophosphorylation in mouse P301L tau knockin neurons. Neurobiol Dis 85:1-10.

Rothwell NJ, Luheshi GN (2000) Interleukin 1 in the brain: biology, pathology and therapeutic target. Trends Neurosci 23:618-625.

Rudiger A, Singer M (2007) Mechanisms of sepsis-induced cardiac dysfunction. Crit Care Med 35:1599-1608.

Sallam MY, El-Gowilly SM, El-Gowelli HM, El-Lakany MA, El-Mas MM (2018) Additive counteraction by $\alpha 7$ and $\alpha 4 \beta 2$-nAChRs of the hypotension and cardiac sympathovagal imbalance evoked by endotoxemia in male rats. Eur J Pharmacol 834:36-44.

Sankowski R, Mader S, Valdés-Ferrer SI (2015) Systemic inflammation and the brain: novel roles of genetic, molecular, and environmental cues as drivers of neurodegeneration. Front Cell Neurosci 9:28.

Schaeffer V, Lavenir I, Ozcelik S, Tolnay M, Winkler DT, Goedert M (2012) Stimulation of autophagy reduces neurodegeneration in a mouse model of human tauopathy. Brain 135:2169-2177.
Sebastián D, Hernández-Alvarez MI, Segalés J, Sorianello E, Muñoz JP, Sala D, Waget A, Liesa M, Paz JC, Gopalacharyulu P, Orešič M, Pich S, Burcelin R, Palacín M, Zorzano A (2012) Mitofusin 2 (Mfn2) links mitochondrial and endoplasmic reticulum function with insulin signaling and is essential for normal glucose homeostasis. Proc Natl Acad Sci U S A 109:5523-5528.

Shaftel SS, Griffin WS, O’Banion MK (2008) The role of interleukin-1 in neuroinflammation and alzheimer disease: an evolving perspective. J Neuroinflammation 5:7.

Sheng JG, Bora SH, Xu G, Borchelt DR, Price DL, Koliatsos VE (2003) Lipopolysaccharide-induced-neuroinflammation increases intracellular accumulation of amyloid precursor protein and amyloid beta peptide in APPswe transgenic mice. Neurobiol Dis 14:133-145.

Sugiura A, Nagashima S, Tokuyama T, Amo T, Matsuki Y, Ishido S, Kudo Y, McBride HM, Fukuda T, Matsushita N, Inatome R, Yanagi S (2013) MITOL regulates endoplasmic reticulum-mitochondria contacts via Mitofusin2. Mol Cell 51:20-34.

Sy M, Kitazawa M, Medeiros R, Whitman L, Cheng D, Lane TE, Laferla FM (2011) Inflammation induced by infection potentiates tau pathological features in transgenic mice. Am J Pathol 178:2811-2822.

Szabadkai G, Simoni AM, Chami M, Wieckowski MR, Youle RJ, Rizzuto R (2004) Drp-1-dependent division of the mitochondrial network blocks intraorganellar $\mathrm{Ca}^{2+}$ waves and protects against $\mathrm{Ca}^{2+}$-mediated apoptosis. Mol Cell 16:59-68.

Tan Y, Ouyang H, Xiao X, Zhong J, Dong M (2019) Irisin ameliorates septic cardiomyopathy via inhibiting DRP1-related mitochondrial fission and normalizing the JNK-LATS2 signaling pathway. Cell Stress and Chaperones 24:595-608.

Vitkovic L, Bockaert J, Jacque C (2000) “Inflammatory” cytokines: neuromodulators in normal brain? J Neurochem 74:457-471.

Wang L, Gao J, Liu J, Siedlak SL, Torres S, Fujioka H, Huntley ML, Jiang Y, Ji H, Yan T, Harland M, Termsarasab P, Zeng S, Jiang Z, Liang J, Perry G, Hoppel C, Zhang C, Li H, Wang X (2018) Mitofusin 2 regulates axonal transport of calpastatin to prevent neuromuscular synaptic elimination in skeletal muscles. Cell Metab 28:400-414.e8.

Wang W, Zhang F, Li L, Tang F, Siedlak SL, Fujioka H, Liu Y, Su B, Pi Y, Wang $\mathrm{X}$ (2015) MFN2 couples glutamate excitotoxicity and mitochondrial dysfunction in motor neurons. J Biol Chem 290:168-182.

Wang X, Su B, Lee HG, Li X, Perry G, Smith MA, Zhu X (2009) Impaired balance of mitochondrial fission and fusion in Alzheimer's disease. J Neurosci 29:9090-9103.

Wang X, Su B, Liu W, He X, Gao Y, Castellani RJ, Perry G, Smith MA, Zhu X (2011) DLP1-dependent mitochondrial fragmentation mediates 1-methyl-4-phenylpyridinium toxicity in neurons: implications for Parkinson's disease. Aging Cell 10:807-823.

Wang X, Petrie TG, Liu Y, Liu J, Fujioka H, Zhu X (2012) Parkinson's disease-associated DJ-1 mutations impair mitochondrial dynamics and cause mitochondrial dysfunction. J Neurochem 121:830-839.

Weber A, Wasiliew P, Kracht M (2010) Interleukin-1 (IL-1) pathway. Sci Signal $3: \mathrm{cm} 1$.

Wilkins HM, Swerdlow RH (2016) Relationships between mitochondria and neuroinflammation: implications for Alzheimer's disease. Curr Top Med Chem 16:849-857.

Yu J, Shi J, Wang D, Dong S, Zhang Y, Wang M, Gong L, Fu Q, Liu D (2016) Heme oxygenase-1/carbon monoxide-regulated mitochondrial dynamic equilibrium contributes to the attenuation of endotoxin-induced acute lung injury in rats and in lipopolysaccharide-activated macrophages. Anesthesiology 125:1190-1201.

Yu T, Robotham JL, Yoon Y (2006) Increased production of reactive oxygen species in hyperglycemic conditions requires dynamic change of mitochondrial morphology. Proc Natl Acad Sci U S A 103:26532658.

Zhao T, Huang X, Han L, Wang X, Cheng H, Zhao Y, Chen Q, Chen J, Cheng H, Xiao R, Zheng M (2012) Central role of mitofusin 2 in autophagosomelysosome fusion in cardiomyocytes. J Biol Chem 287:23615-23625.

Zujovic V, Benavides J, Vigé X, Carter C, Taupin V (2000) Fractalkine modulates TNF-alpha secretion and neurotoxicity induced by microglial activation. Glia 29:305-315.

Zujovic V, Schussler N, Jourdain D, Duverger D, Taupin V (2001) In vivo neutralization of endogenous brain fractalkine increases hippocampal TNFalpha and 8-isoprostane production induced by intracerebroventricular injection of LPS. J Neuroimmunol 115:135-143. 